\title{
A missense mutation in TUBD1 is associated with high juvenile mortality in Braunvieh and Fleckvieh cattle
}

Hermann Schwarzenbacher ${ }^{1}$, Johann Burgstaller ${ }^{2}$, Franz R. Seefried ${ }^{3}$, Christine Wurmser ${ }^{4}$, Monika Hilbe ${ }^{5}$, Simone Jung ${ }^{4}$, Christian Fuerst ${ }^{1}$, Nora Dinhopl ${ }^{6}$, Herbert Weissenböck ${ }^{6}$, Birgit Fuerst-Waltt ${ }^{7}$, Marlies Dolezal ${ }^{8}$, Reinhard Winkler ${ }^{9}$, Oskar Grueter ${ }^{10}$, Ulrich Bleul ${ }^{11}$, Thomas Wittek ${ }^{2}$, Ruedi Fries ${ }^{4}$ and Hubert Pausch ${ }^{4 *}$

\begin{abstract}
Background: Haplotypes with reduced or missing homozygosity may harbor deleterious alleles that compromise juvenile survival. A scan for homozygous haplotype deficiency revealed a short segment on bovine chromosome 19 (Braunvieh haplotype 2, BH2) that was associated with high juvenile mortality in Braunvieh cattle. However, the molecular genetic underpinnings and the pathophysiology of $\mathrm{BH} 2$ remain to be elucidated.

Results: The frequency of $\mathrm{BH} 2$ was $6.5 \%$ in 8,446 Braunvieh animals from the national bovine genome databases. Both perinatal and juvenile mortality of $\mathrm{BH} 2$ homozygous calves were higher than the average in Braunvieh cattle resulting in a depletion of $\mathrm{BH} 2$ homozygous adult animals $\left(P=9.3 \times 10^{-12}\right)$. The analysis of whole-genome sequence data from 54 Braunvieh animals uncovered a missense mutation in TUBD1 (rs383232842, p.H210R) that was compatible with recessive inheritance of $\mathrm{BH} 2$. The availability of sequence data of 236 animals from diverse bovine populations revealed that the missense mutation also segregated at a low frequency $(1.7 \%)$ in the Fleckvieh breed. A validation study in 37,314 Fleckvieh animals confirmed high juvenile mortality of homozygous calves $\left(P=2.2 \times 10^{-15}\right)$. Our findings show that the putative disease allele is located on an ancestral haplotype that segregates in Braunvieh and Fleckvieh cattle. To unravel the pathophysiology of $\mathrm{BH} 2$, six homozygous animals were examined at the animal clinic. Clinical and pathological findings revealed that homozygous calves suffered from chronic airway disease possibly resulting from defective cilia in the respiratory tract.
\end{abstract}

Conclusions: A missense mutation in TUBD1 is associated with high perinatal and juvenile mortality in Braunvieh and Fleckvieh cattle. The mutation is located on a common haplotype likely originating from an ancient ancestor of Braunvieh and Fleckvieh cattle. Our findings demonstrate for the first time that deleterious alleles may segregate across closed cattle breeds without recent admixture. Homozygous calves suffer from chronic airway disease resulting in poor growth performance and high juvenile mortality. The respiratory manifestations resemble key features of diseases resulting from impaired function of airway cilia.

Keywords: Braunvieh haplotype 2, Juvenile mortality, Tubulin delta 1, Primary ciliary dyskinesia, Ciliopathy, Chronic respiratory disease

\footnotetext{
* Correspondence: hubert.pausch@tierzucht.tum.de

${ }^{4}$ Lehrstuhl fuer Tierzucht, Technische Universitaet Muenchen, Freising 85354,

Germany

Full list of author information is available at the end of the article
} 


\section{Background}

Reducing calf mortality is an important objective in cattle breeding populations both for animal welfare and economic reasons. Bovine calf losses including late abortions, stillbirths and diseases during rearing may be in excess of $10 \%$ [1-4]. Most rearing losses are attributable to infectious diarrheal and respiratory diseases [5]. While a number of genetic variants predisposing to high perinatal mortality have been identified in bovine populations (e.g., [6, 7]), the mapping of loci affecting disease susceptibility and rearing success is difficult because of their low heritability $[8,9]$.

Case-control association testing using genome-wide marker data facilitated the discovery of causal variants for monogenic disorders that result in high juvenile mortality [10-16]. Although the pathophysiology of such conditions is heterogeneous, pertinently affected calves may be apathetic, without vigor, retarded in growth or highly susceptible to infectious disease. Affected calves may be born without typical signs of disease but develop clinical features shortly after birth $[10,11,14]$.

The availability of large-scale genotype data enables the identification of haplotypes with homozygosity depletion $[14,17,18]$. Haplotypes with reduced or missing homozygosity in adult animals are likely to harbor recessive alleles that are associated with an increased pre-, peri- or postnatal mortality. Genome-wide scans for homozygous haplotype deficiency in North American [17] and European Braunvieh [19] populations uncovered a short segment on bovine chromosome 19 that was associated with high postnatal calf losses (OMIA 001939-9913). The segment associated with calf mortality was denominated 424.49 and BTA19-1 in the North American [17] and European Braunvieh [19] populations, respectively. Since 2013, the associated genomic segment is consistently referred to as $\mathrm{BH} 2$ with $\mathrm{BH}$ being an abbreviation for Braunvieh haplotype [20].

In the present study, we exploit comprehensive genotyping and sequencing data to detect a missense mutation in TUBD1 that is most likely causal for the high juvenile mortality of $\mathrm{BH} 2$ homozygous calves. We validate the missense mutation in an independent cattle population and provide evidence that homozygous calves suffer from chronic respiratory disease.

\section{Results}

\section{$\mathrm{BH} 2$ compromises juvenile survival in Braunvieh cattle}

We exploited array-derived genotypes of 8,446 Braunvieh animals from the national bovine genome databases to scan for homozygosity depletion on chromosome 19. Thirty-two haplotypes in strong linkage disequilibrium that were located between $3.67 \mathrm{Mb}$ and $13.38 \mathrm{Mb}$ on BTA19 showed a significant depletion of homozygous animals $\left(P<1 \times 10^{-4}\right)$. The length of the haplotypes with homozygosity depletion ranged from 0.54 to $6.33 \mathrm{Mb}$.
Harmful effects on fertility and calf survival were analyzed for all haplotypes with homozygosity depletion using logistic regression analyses with insemination and rearing success as response variables. None of the haplotypes tested was associated with insemination success $(P>0.01)$ ruling out homozygous haplotype deficiency to result from early embryonic losses. However, all haplotypes were associated with high juvenile mortality $\left(P<3.2 \times 10^{-9}\right)$. After combining the $\mathrm{P}$ values from homozygosity depletion and calf survival analyses, the most significantly associated haplotype (hereafter referred to as $\mathrm{BH} 2$ ) was located within a 1.14 Mb interval between 10,694,269 bp and 11,833,182 bp on BTA19 (Fig. 1a). The frequency of $\mathrm{BH} 2$ was $6.5 \%$. Only one animal was homozygous although 41 were expected $\left(P=9.3 \times 10^{-12}\right)$. The analysis of 32,100 calving records revealed that the first-year mortality of descendants from $\mathrm{BH} 2$ risk-matings was increased by $4.6 \%$ compared to non-risk matings $(P=1.8 \times 10$ ${ }^{-28}$ ) (Table 1).

Two male Braunvieh calves homozygous for $\mathrm{BH} 2$ were detected in the Swiss bovine genome database. According to the possessing farmers, both calves were underweight at birth. One calf died for an unknown cause at 72 days of age and was not available for phenotyping. The second calf $\left(\mathrm{BH}_{2}\right.$ hom $)$ was referred to the animal clinic at 66 days of age. During a hospitalization period of 95 days, $\mathrm{BH} 2$ hom suffered repeatedly from bronchopneumonia. At 161 days of age, $\mathrm{BH} 2_{\text {hom }}$ was euthanized because of suddenly occurring severe dyspnea.

\section{A missense mutation in TUBD1 is compatible with recessive inheritance of $\mathrm{BH} 2$}

To identify the causal mutation for the high mortality of $\mathrm{BH} 2$ homozygous calves, we analyzed re-sequencing data of $\mathrm{BH} 22_{\text {hom }}$, five heterozygous $\mathrm{BH} 2$ carriers and 48 control animals of the Braunvieh population. The average genome coverage in $\mathrm{BH} 2_{\mathrm{hom}}$, five heterozygous carriers and 48 control animals, respectively, was 17.36, $12.08 \pm$ 1.39 and $11.46 \pm 1.54$ fold. Multi-sample variant calling in 54 sequenced animals yielded genotypes for 99,797 single nucleotide and short insertion and deletion polymorphisms and 10,497 structural variants located within the 9.71 Mb interval (from 3.67 Mb to $13.38 \mathrm{Mb}$ ) with homozygosity depletion encompassing BH2. These 110,294 polymorphic sites were filtered for variants that were compatible with recessive inheritance of $\mathrm{BH} 2$ that is homozygous for the non-reference allele in $\mathrm{BH} 2_{\text {hom }}$, heterozygous in $\mathrm{BH} 2$ carriers and homozygous for the reference allele in control animals. This filtering revealed 52 variants in $\mathrm{LD}$ with $\mathrm{BH} 2$ that were located between 9,428,803 bp and 11,811,557 bp on BTA19 (Additional file $1)$ : fifty variants were located in non-coding regions, one variant (rs479748045) was a synonymous mutation in the PPM1E (protein phosphatase, $M g 2+/ M n 2+$ dependent $1 E$ ) 


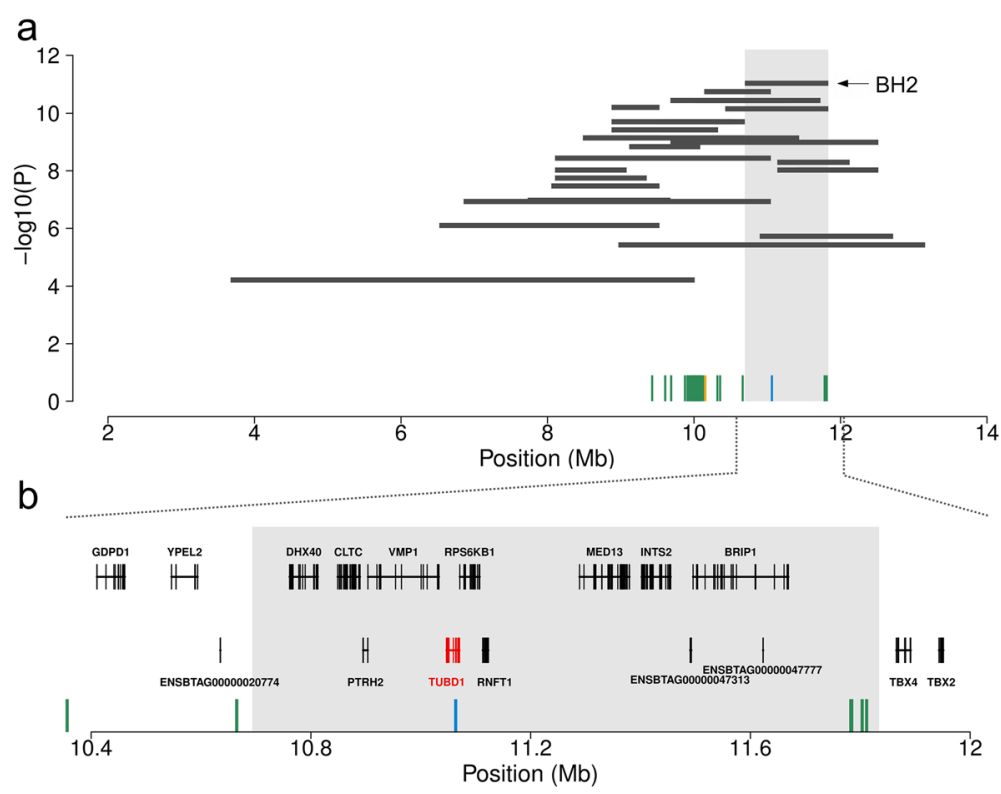

Fig. 1 Schematic representation of the proximal region of bovine chromosome 19 encompassing BH2. Association of 32 haplotypes with homozygosity depletion and postnatal calf mortality (a). Each horizontal bar represents a haplotype with homozygosity depletion. The grey shaded area highlights the most significantly associated haplotype (BH2). Green, orange and blue vertical bars represent 50 non-coding variants, one synonymous variant in PPM1E and one missense variant in TUBD1, respectively. Genes located within the BH2 interval (b). The grey shaded area highlights the position of $\mathrm{BH} 2$. Green and blue vertical bars represent six non-coding variants and the missense variant in the TUBD1 gene

gene and one variant (rs383232842) was a missense mutation in the TUBD1 (tubulin delta 1) gene (Additional file 2). Of the 52 variants in LD with BH2 four non-coding variants and the missense mutation in TUBD1 were located within the $1.14 \mathrm{Mb}$ interval (BH2) that showed the strongest association with homozygosity depletion and postnatal calf mortality (Fig. 1b). The four non-coding variants that were located within the $\mathrm{BH} 2$ interval were more than $50 \mathrm{~kb}$ away from coding sequences. Moreover, two of them (rs385391620 at 11,782,742 bp and rs386039720 at $11,803,361 \mathrm{bp}$ ) were found to occur in homozygous state among 1682 animals from various bovine breeds that had been sequenced for the 1000 bull genomes project (Additional file 1) [21] and are thus less likely to be causal for the high postnatal mortality associated with homozygosity for $\mathrm{BH} 2$. In conclusion, the rs 383232842 mutation in the TUBD1 coding region and two non-coding variants were considered as candidate causal variants for $\mathrm{BH} 2$ (Additional file 3). The rs383232842 C-allele causes a substitution of a histidine by an arginine at a conserved position in tubulin delta 1 (ENSBTAP00000001700.5:p.H210R) (Additional file 4). The amino acid substitution is predicted to be damaging to protein function (SIFT-score: 0.03, Polyphen-score: 0.23). We obtained genotypes of the rs383232842 polymorphism in 661 adult Braunvieh animals using a KASP genotyping assay; the mutation was in high linkage disequilibrium (LD) with $\mathrm{BH} 2\left(\mathrm{r}^{2}=0.98\right)$ and none of 661 genotyped animals was homozygous for the C-allele (Table 2). Genotypes at rs383232842 differed from the haplotype-based $\mathrm{BH} 2$ states for five (out of 661) animals possibly because of haplotype recombination or phasing errors. The rs383232842 polymorphism was in LD with the two non-coding variants (Additional files 1 and 5).

\section{The $\mathrm{BH} 2$ haplotype affects rearing success in another cattle breed}

The analysis of whole-genome sequencing data from 236 animals from six bovine breeds other than Braunvieh

Table 1 Harmful effects of two haplotypes with reduced homozygosity in Braunvieh (BH2) and Fleckvieh ( $\left.\mathrm{BH} 2_{\mathrm{Fv}}\right)$ cattle

\begin{tabular}{|c|c|c|c|c|c|c|c|c|c|c|}
\hline \multirow[t]{2}{*}{ Haplotype } & \multirow[t]{2}{*}{ Chr } & \multicolumn{2}{|l|}{ Position } & \multirow{2}{*}{$\begin{array}{l}\text { Haplotype } \\
\text { frequency (\%) }\end{array}$} & \multicolumn{2}{|c|}{ Number of calvings } & \multicolumn{4}{|c|}{ Difference in survival rate } \\
\hline & & Start (bp) & Stop (bp) & & risk & non-risk & day 2 & day 10 & day 365 & $P$ \\
\hline$\overline{\mathrm{BH}} 2$ & 19 & $10,694,269$ & $11,833,182$ & 6.5 & 6,299 & 25,801 & -2.1 & -3.9 & -4.6 & $1.8 \times 10^{-28}$ \\
\hline $\mathrm{BH} 2_{\mathrm{FV}}$ & 19 & $10,406,017$ & $11,453,904$ & 1.7 & 1,296 & 45,270 & -4.0 & -4.0 & -6.1 & $2.2 \times 10^{-15}$ \\
\hline
\end{tabular}

The juvenile mortality of calves from risk matings was compared to non-risk matings. Matings between carrier bulls and cows descending from carrier bulls were considered as risk matings and matings between non-carrier bulls and cows descending from carrier bulls were considered as non-risk matings. The difference in survival rate refers to the proportion of additional calf losses in risk compared to non-risk matings. The physical position of the haplotypes was based on the UMD3.1 assembly of the bovine genome 
Table 2 Genotypes for a missense mutation in TUBD1 in 661 adult Braunvieh cattle

\begin{tabular}{lllll}
\hline $\begin{array}{l}\text { BH2 haplotype } \\
\text { status }\end{array}$ & $\mathrm{N}$ & \multicolumn{4}{l}{ Genotype at rs383232842 } \\
\cline { 3 - 5 } & & 220 & $\mathrm{~T} / \mathrm{T}$ & $\mathrm{C} / \mathrm{C}$ \\
\hline Non-carrier & 221 & 220 & - \\
Carrier & 440 & 4 & 436 & -
\end{tabular}

Genotypes for the rs383232842 polymorphism were obtained in 661 Braunvieh animals using KASP genotyping assays. The haplotype status (non-carrier, carrier) of the animals was determined using phased genotypes obtained with the Illumina BovineSNP50 array. Note that the genotyped animals are not representative for the entire Braunvieh population because we preferentially selected heterozygous $\mathrm{BH} 2$ haplotype carriers for genotyping

revealed that rs383232842 was heterozygous in seven out of 149 Fleckvieh animals (Additional file 1 and 3). To obtain genotypes for rs383232842 in a representative sample of the Fleckvieh population, we genotyped 3807 randomly selected adult animals using a KASP genotyping assay. Among those, 142 were heterozygous and none was homozygous for the $\mathrm{C}$-allele corresponding to a C-allele frequency of $1.86 \%$. Of 3807 animals that had been genotyped at rs383232842, 1,974 were also genotyped with the Illumina BovineHD Bead chip comprising 777,962 SNPs. The availability of dense marker data enabled us to detect an $1.05 \mathrm{Mb}$ haplotype $\left(\mathrm{BH} 2_{\mathrm{FV}}\right.$; from $10,406,017 \mathrm{bp}$ to $11,453,904 \mathrm{bp}$ ) that was specific for the rs383232842 C-allele: ninety-two animals that carried the $\mathrm{C}$-allele were heterozygous carriers of $\mathrm{BH} 2_{\mathrm{FV}}$, whereas 1882 animals that were homozygous for the reference allele did not carry $\mathrm{BH} 2_{\mathrm{FV}}$. In 37,314 Fleckvieh animals of the national bovine genome databases that had been genotyped with the Illumina BovineSNP50 Bead chip, the frequency of $\mathrm{BH} 2$ FV was $1.68 \%$. Only four animals were homozygous for $\mathrm{BH} 2_{\mathrm{FV}}$ although ten were expected $(P=0.09)$. The analysis of 46,566 calving records revealed that the first-year mortality of descendants from $\mathrm{BH} 2_{\mathrm{FV}}$ risk matings was increased by $6.1 \%$ compared to progeny from non-risk matings $\left(P=2.2 \times 10^{-15}\right)$ (Table 1$)$.

\section{A common haplotype with the rs383232842 C-allele segregates in Braunvieh and Fleckvieh cattle}

BH2 encompasses a 1.14 Mb segment from 10,694,269 bp to $11,833,182 \mathrm{bp}$. It is defined by eleven SNPs of the Illumina BovineSNP50 Bead chip. BH2 $2_{\mathrm{FV}}$, defined by 249 SNPs of the Illumina BovineHD Bead chip, is located between 10,406,017 bp and 11,453,904 bp. The BH2 alleles are a subset of the $\mathrm{BH} 2 \mathrm{FV}$ alleles within a $741 \mathrm{~kb}$ segment between 10,694,269 bp and 11,435,269 bp (Additional file 6). Since both $\mathrm{BH} 2$ and $\mathrm{BH} 2{ }_{\mathrm{FV}}$ contain the rs383232842 C-allele, a common origin of the two haplotypes is most likely. The analysis of pedigree records enabled us to track $\mathrm{BH} 2$ and $\mathrm{BH} 2_{\mathrm{FV}}$ back to the Braunvieh bull Rancho Rustic My Design (birth year 1963) and the Fleckvieh bull Polzer (birth year 1959), respectively. However, we were not able to identify common ancestors in their pedigrees possibly because of missing pedigree information from very distantly related relatives. Cluster analyses using genome-wide marker data of 442 Fleckvieh and 280 Braunvieh animals born between 1970 and 2010 revealed no evidence for an exchange of genetic material between both breeds (Additional file 7).

\section{$\mathrm{BH} 2$ segregates in the Holstein breed, but does not contain the rs383232842 C-allele}

To test if the common $\mathrm{BH} 2 / \mathrm{BH} 2_{\mathrm{FV}}$ haplotype segment segregated in another dairy breed, we analyzed haplotypes of 8840 adult Holstein animals that had been genotyped with the Illumina BovineSNP50 Bead chip. We identified 917 heterozygotes and 30 homozygotes for $\mathrm{BH} 2$ among the genotyped Holstein animals. The observed haplotype distribution did not deviate from the Hardy-Weinberg equilibrium $(P=0.54)$. We additionally genotyped the rs383232842 polymorphism in 503 Holstein bulls using a KASP genotyping assay. All of them including 74 heterozygotes and two homozygotes for $\mathrm{BH} 2$ were homozygous for the rs383232842 reference allele (Additional file 6). Another 311 Holstein animals that had been sequenced within the 1000 bull genomes project [21] were homozygous for the reference allele indicating that the rs383232842 C-allele does not segregate in this breed. The non-reference alleles at two non-coding variants located within the $\mathrm{BH} 2$ interval were only observed at very low frequency in Holstein cattle (Additional file 1 \& Additional file 5).

\section{Identification of $\mathrm{BH} 2$ homozygous calves}

To unravel the pathophysiology associated with $\mathrm{BH} 2$, we initiated a monitoring project in the Austrian and Swiss Braunvieh populations. Breeding consultants collected ear tissue samples of calves descending from $\mathrm{BH} 2$ risk matings immediately after birth for genetic investigations. Of 117 genotyped calves, twelve were homozygous for the rs383232842 C-allele (Table 3). Among those, eight were stillborn or died shortly after birth. All stillborn homozygotes were underweight at 25 to $30 \mathrm{~kg}$ and appeared underdeveloped although gestation length was normal (Additional file 8). Apart from low body weight, necropsy revealed no signs of disease. Four live born homozygous calves (BV1-BV4) were referred to the animal clinic.

Table 3 Sampling of calves from $\mathrm{BH} 2$ risk matings

\begin{tabular}{lllll}
\hline $\begin{array}{l}\text { Genotype at } \\
\text { rs383232842 }\end{array}$ & $\begin{array}{l}\text { Number of } \\
\text { calves }\end{array}$ & Stillborn & $\begin{array}{l}\text { Died within } \\
10 \text { days }\end{array}$ & $\begin{array}{l}\text { Died within } \\
30 \text { days }\end{array}$ \\
\hline$\pi$ & 45 & 1 & 0 & 1 \\
$C T$ & 60 & 4 & 1 & 0 \\
$C C$ & 12 & 5 & 0 & 3 \\
\hline
\end{tabular}

Genotypes of 117 calves descending from $\mathrm{BH} 2$ risk matings. Note the high perinatal mortality $(8 / 12)$ of calves homozygous for the rs383232842 C-allele 
During the sampling of $\mathrm{BH} 2$ homozygous calves, we encountered a male Fleckvieh calf (FV1) with low birth weight and postnatal growth restriction that descended from a $\mathrm{BH} 2_{\mathrm{FV}}$ carrier bull. At 40 days of age, its weight was $28.5 \mathrm{~kg}$, which is only a third compared to healthy Fleckvieh calves of the same age. FV1 was tested negatively for two recessive mutations that are known to cause retarded growth [14, 22]. Another 16-months old Braunvieh bull (BV5) with poor growth performance and a history of recurrent respiratory disease was reported by a farmer. Inspection of the bull's pedigree revealed $\mathrm{BH} 2$ carriers among its paternal and maternal ancestors. Sanger sequencing of DNA samples from FV1 and BV5 confirmed that both animals were homozygous for the rs383232842 C-allele. Both animals were referred to the animal clinic.

\section{Homozygous animals suffer from chronic airway disease}

At admission, six homozygous animals were emaciated and their heads appeared elongated (Fig. 2, Additional file 9). Initial examinations revealed aberrant breathing sounds (i.e., enforced vesicular breathing, rhonchus, coughing), tachypnea, tachycardia and excessive mucous exudation from the nostrils (Table 4). The analysis of blood parameters revealed iron deficiency and increased monocyte counts in some animals possibly indicating ongoing response to infectious disease (Additional file 10). The clinical features led to the diagnosis of bronchopneumonia and the animals were treated accordingly. Although the medication alleviated the clinical symptoms, respiratory disease recurred repeatedly. Due to the steadily declining health condition with no prospect for improvement, all animals were euthanized and subjected to necropsy.

At necropsy, the animals were underweight and emaciated. Macroscopic abnormalities of the respiratory tract such as mucopurulent rhinitis, tracheobronchitis and lung lesions became evident in all animals (Additional file 11). Histological sections of tissue samples from the upper and lower respiratory tract revealed hyperplastic ciliated epithelia with intraepithelial neutrophils and bronchus-associated lymphoid tissue (BALT) hyperplasia, respectively, accompanied by bronchiolitis and bronchiectasis (Additional file 12). The lumen of bronchi and bronchioles were obstructed by mucopurulent exudate and the surrounding lung tissue was atelectatic and showed minimal intra-alveolar infiltration of inflammatory cells. Transmission electron microscopic (TEM)

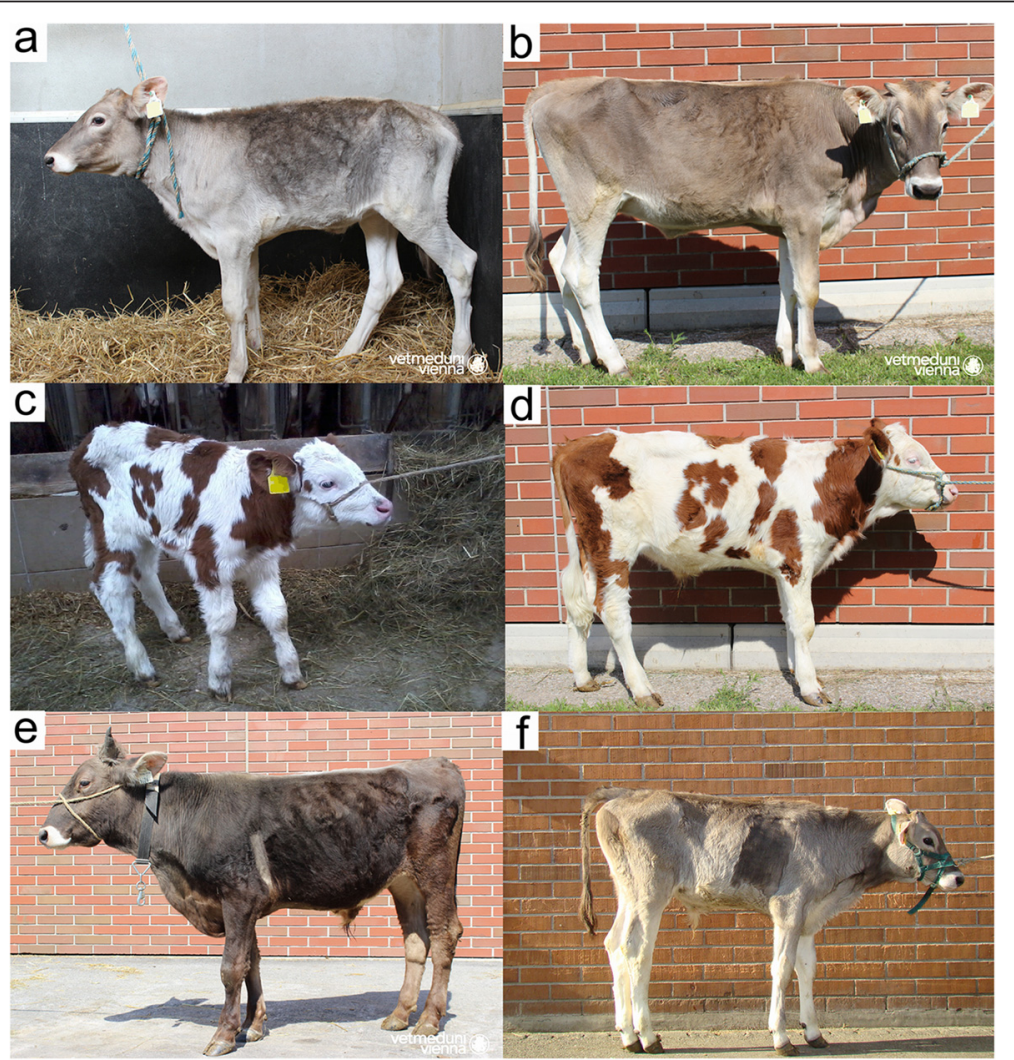

Fig. 2 Phenotypic manifestation of homozygosity for BH2 in four animals. Pictures of BV2 (a, b), FV1 (c, d), BV5 (e) and BV4 (f) were taken at the time of admission in the animal clinic $(\mathbf{a}, \mathbf{c})$ and shortly before euthanasia $(\mathbf{b}, \mathbf{d}, \mathbf{e}, \mathbf{f})$, respectively. A detailed description of the disease manifestations is available in Table 4 
Table 4 Characteristics of six hospitalized animals

\begin{tabular}{lllllll}
\hline Parameter & BV1 & BV2 & FV1 & BV3 & BV4 & BV5 \\
\hline Breed & Braunvieh & Braunvieh & Fleckvieh & Braunvieh & Braunvieh & Braunvieh \\
Sex & male & female & male & male & male & male \\
Age at admission (days) & 41 & 44 & 75 & 13 & 1 & 485 \\
Weight at admission (kg) & 58 & 59 & 71 & 31 & 33 & 227 \\
Pulse (heartbeats per minute) & 100 & 104 & 100 & 140 & 138 & 60 \\
Body temperature $\left({ }^{\circ} \mathrm{C}\right)$ & 38.3 & 39.9 & 39.2 & 38.6 & 38.3 & 39.1 \\
Respiratory rate (breaths per minute) & 40 & 48 & 40 & 40 & 54 & 60 \\
BCS (score) & 2.5 & 2 & 2.5 & 2 & 2 & 1.5 \\
Auscultation lungs & + vesicular & ++ vesicular & ++ vesicular & +++ vesicular & rhonchus & +++ vesicular-bronchial \\
Hospitalization period (days) & 109 & 164 & 111 & 19 & 157 & 17 \\
Age at euthanasia (days) & 150 & 208 & 186 & 32 & 158 & 502 \\
Weight at euthanasia (kg) & 168 & 151.5 & 106.5 & 31 & 134 & 258 \\
\hline
\end{tabular}

Parameters from the initial examination of six Braunvieh (BV1-BV5) and Fleckvieh (FV1) animals homozygous for the rs383232842 C-allele

data of ciliated epithelial tissue were available from the upper and lower respiratory tract of four animals (FV1, BV1, BV2, BV4). Analyses of TEM sections revealed defective microtubule organization in 20-30 \% of ciliary cross-sections including transposition faults, microtubular disorganization, absence of outer and central microtubule pair and aberrations of the outer and inner dynein arms (Fig. 3).

\section{Homozygosity for $\mathrm{BH} 2$ may be incompletely penetrant} Breeding consultants reported another three Braunvieh animals that were homozygous for $\mathrm{BH} 2$. These homozygous animals were detected during routine genomic evaluation using genotypes of the Illumina BovineSNP50 Bead chip. Sanger sequencing of DNA samples confirmed homozygosity for the rs383232842 C-allele in all animals. While one young bull (BV6) was healthy and normally developed at the age of 370 days, two animals (BV7, BV8) were retarded in growth and had a history of recurrent respiratory disease (Additional file 13). The three homozygous animals were also homozygous for the alternate allele at two noncoding variants in LD with rs383232842 (Additional file 5).

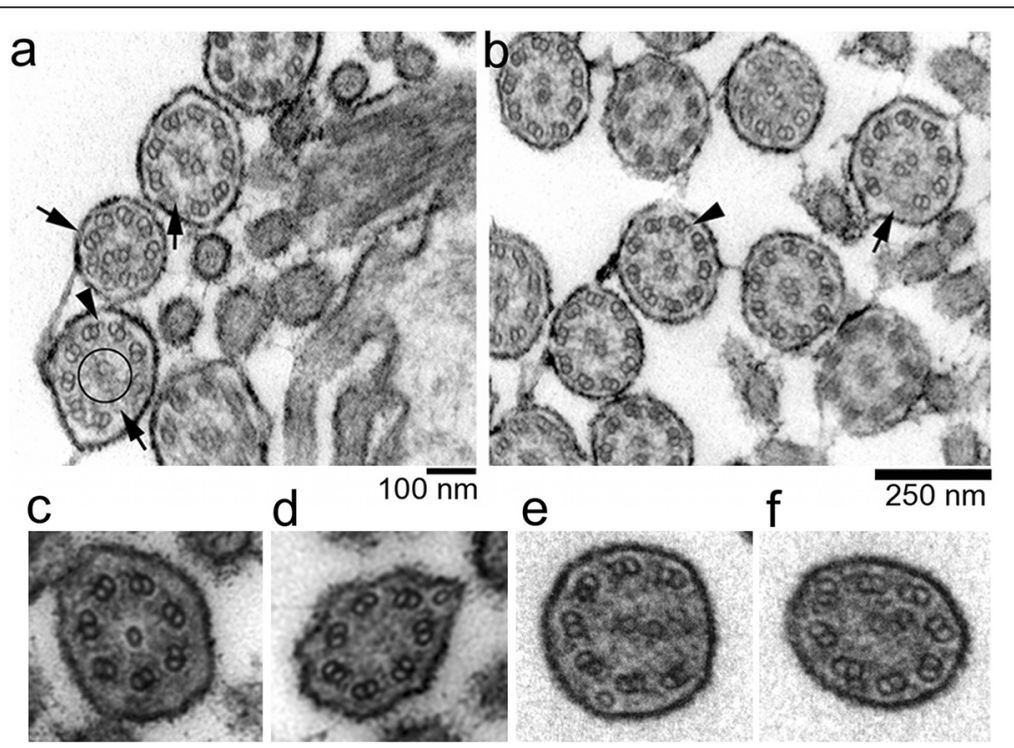

Fig. 3 Transmission electron microscopy of respiratory cilia of BH2 homozygous animals. Motile cilia are characterized by a typical "9x2 + 2" architecture, i.e., nine outer microtubule doublets surrounding a central pair of microtubule. The TEM sections of respiratory cilia of bronchi from BV1 (a) and FV1 (b) revealed multiple ultrastructural defects including transposition defects of microtubuli and microtubular disorganization (arrows), absence of a central microtubule pair (circle), and loss of inner and/or outer dynein arms (arrowheads). Magnification of cilia with typical ultrastructural defects found in the respiratory tract of BV5 (c, d) and BV4 (e, f) 


\section{Discussion}

We show that the proximal region of bovine chromosome 19 is associated with high peri- and postnatal calf losses in Braunvieh cattle, corroborating previous reports of a harmful haplotype (Braunvieh haplotype 2, BH2) in European and North-American Braunvieh populations $[17,19]$. The joint analysis of homozygosity depletion and insemination and rearing data revealed a $1.14 \mathrm{Mb}$ segment between 10,694,269 bp and $11,833,182$ bp on BTA19 as the most likely interval harboring the causal mutation, which agrees with previous findings $[17,19]$. The first-year mortality of calves descending from $\mathrm{BH} 2$ risk matings is less than expected for a lethal recessive disorder, indicating that some $\mathrm{BH} 2$ homozygous calves may reach adulthood. Despite its detrimental effect on juvenile survival, the frequency of $\mathrm{BH} 2$ is high in the European Braunvieh populations (6.5 \%). Based on the retrospective analysis of inadvertent $\mathrm{BH} 2$ riskmatings that happened in the Austrian Braunvieh population ( $N=50,000$ calvings per year) between 1990 and 2013, we estimated that between 11 and $393 \mathrm{BH} 2$ homozygous animals were born annually in the past 25 years. Deleterious alleles may reach high frequency in cattle populations because of the widespread use of unnoticed carrier bulls in artificial insemination and pleiotropic effects on desirable traits [23-25]. So far, there is no evidence that $\mathrm{BH} 2$ has desirable effects on important breeding objectives. Several harmful conditions other than $\mathrm{BH} 2$ segregate in the Braunvieh populations [17, 26-29]. Pinpointing the causal mutation allows for the implementation of efficient genome-based mating programs to avoid the inadvertent mating of carrier animals while maintaining genetic diversity and high rates of genetic gain. A missense mutation (rs383232842, p.H210R) in TUBD1 was found to be compatible with recessive inheritance of $\mathrm{BH} 2$. Affected, carrier and noncarrier animals were homozygous for the alternate allele, heterozygous and homozygous, respectively, for the reference allele at rs383232842. Although $\mathrm{BH} 2$ is in high LD with rs383232842, the identification of carrier animals is less reliable with haplotype information. Five out of 661 animals were misclassified based on the haplotype test indicating both lower specificity and sensitivity of haplotype-based classification of animals into carriers and non-carriers which agrees with previous findings in cattle $[10,18,26,30]$.

Pinpointing causal mutations is often difficult as many sequence variants may be compatible with the presumed pattern of inheritance (e.g., $[18,24])$. In 54 sequenced Braunvieh animals of our study, two intergenic variants were in complete LD with rs383232842. Yet we consider rs383232842 as the most likely to be causal because it results in a substitution of a conserved amino acid residue. Moreover, all compatible non-coding variants are far away from annotated transcripts and are thus less likely to be functionally important. However, it cannot be ruled out a priori that such variants affect gene regulation, e.g., if located in a distant enhancer region. A criterion for causality is often that the segregation of the candidate variant is restricted to the population affected by the condition (e.g., $[13,14,29,31])$. Strictly applying this criterion to our data would have led to the exclusion of rs383232842 as a plausible causal mutation for BH2, because it segregates in Fleckvieh cattle where a previous scan for homozygous haplotype deficiency did not detect homozygosity depletion at the $\mathrm{BH} 2$ region [14]. In the present study, we specifically tested the $\mathrm{BH} 2_{\mathrm{FV}}$ haplotype with the rs383232842 C-allele for association with juvenile mortality in more than 37,000 Fleckvieh animals. Homozygosity depletion at $\mathrm{BH} 2_{\mathrm{FV}}$ was not significant $(P=0.09)$ possibly because most Fleckvieh animals of the present study were genotyped shortly after birth, before typical signs of disease may become manifest. However, the first-year mortality of descendants is significantly higher in $\mathrm{BH} 2_{\mathrm{FV}}$ risk than non-risk matings $(P=$ $\left.2.2 \times 10^{-15}\right)$ corroborating a detrimental effect of $\mathrm{BH} 2 \mathrm{Fv}$.

The exchange of genetic material between breeds may result in the manifestation of the same recessively inherited conditions in different populations [27]. Kumar Kadri et al. identified a sequence variant that compromises fertility in three admixed Nordic dairy breeds [25]. Drögemüller et al. mapped a sequence variant for an autosomal recessive skin disease in two geographically separated albeit genetically related dog populations [31]. A diagnostic haplotype associated with progressive degenerative myeloencephalopathy (Weaver syndrome) in Braunvieh cattle also segregates in the Holstein cattle breed [32]. However, the most likely causal variant for Weaver syndrome was never found in haplotype carriers from the Holstein cattle breed indicating that an ancestral version of the haplotype without the Weaver mutation persists in Holstein cattle [32,33]. The discovery of a recessive mutation that compromises juvenile survival in Fleckvieh and Braunvieh cattle demonstrates for the first time, that deleterious alleles may segregate across closed cattle populations, e.g., populations without recent admixture. Fleckvieh and Braunvieh animals with the rs383232842 C-allele share a common haplotype, indicating they inherited the corresponding chromosome segment from a common ancestor. However, our data neither revealed common ancestors nor provided evidence of an exchange of genetic material between both breeds. A version of the ancestral haplotype without the rs383232842 C-allele also segregates in Holstein cattle at relatively high frequency $(5.5 \%)$. These findings indicate that the rs383232842 mutation occurred in an ancient ancestor of Braunvieh and Fleckvieh cattle, however, after the divergence from Holstein cattle, which is 
compatible with the history of those breeds [34]. While $\mathrm{BH} 2$ and $\mathrm{BH} 2_{\mathrm{FV}}$ affect juvenile survival in Braunvieh and Fleckvieh cattle, respectively, the haplotype version without the rs383232842 C-allele does not exhibit homozygous haplotype deficiency in Holstein cattle supporting causality of the rs383232842 polymorphism.

To investigate the pathophysiology that may result from the rs383232842 mutation in TUBD1, we examined six homozygous animals. Although the age of the examined animals differed, the disease manifestation was homogeneous: recurrent airway disease resulted in a steadily declining general condition and poor growth performance despite excellent husbandry conditions and medical treatment at the animal clinic. Thus, a more severe disease progression in homozygous animals kept under normal husbandry conditions in conventional farms is likely. The respiratory manifestations resemble disease patterns arising from defective cilia of the respiratory tract [35-37]. We observed microtubular assembly defects in $20-30 \%$ of the cilia of the respiratory epithelium in affected animals which is 4-6 times higher than in healthy individuals [38-40]. Ultrastructural defects of the cilia might also result from infectious or inflammatory diseases [41]. Persistent coughing, tachypnea and airway mucus obstruction in homozygous animals might indicate an impaired mucociliary clearance due to an aberrant ciliary beat pattern [42-45]. We used a cytology brush to collect viable epithelial cells from the nasal mucosa of affected calves to investigate ciliary beat pattern and beat frequency (data not shown). However, all collected samples were excessively covered with mucous exudate and contained numerous inflammatory cells, precluding the analysis of ciliary beating using standard protocols.

Several sequence variants associated with ultrastructural aberrations of respiratory cilia cause chronic airway disease in humans, mice and dogs (e.g., [37, 46, 47]). Respiratory cilia are characterized by a " $9 \times 2+2$ " architecture consisting of a central pair of microtubule surrounded by nine outer microtubule doublets. The basal body is located at the base of the cilium where it attaches to the cell body. Tubulin delta 1 is required for proper microtubule polymerization in basal bodies [4851]. A lack of TUBD1 causes ultrastructural microtubular defects in basal bodies in Chlamydomonas and Paramecium [48, 50, 51]. We also observed disorganized microtubules in airway cilia of animals homozygous for the rs383232842 mutation corroborating a crucial role of TUBD1 for proper assembly of cilia. To our knowledge, this is the first report of a phenotypic effect associated with genomic variation in TUBD1 in a mammalian species. A conserved histidine residue at amino acid position 210 in TUBD1 is involved in microtubule polymerization [49]. Since the rs383232842 C-allele introduces a putatively damaging substitution of the histidine by an arginine, proper microtubule polymerization might be compromised in homozygous animals, resulting in ultrastructural defects of the airway cilia. Besides being involved in cilia assembly, microtubules constitute the bipolar spindle that binds and moves the chromosomes during the different meiotic phases. Disturbed microtubule formation may lead to erroneous segregation of chromosomes with negative effects on growth and differentiation (e.g., [52]). Such disturbances could be responsible for frequently observed prenatal growth retardation and stillbirth of fetuses from $\mathrm{BH} 2$ risk matings.

Most homozygous calves (75\%) were stillborn or died shortly after birth. Six hospitalized calves suffered from airway disease early in life. The general condition of all hospitalized animals declined steadily because the disease was not responsive to medical treatment. However, homozygosity for the rs383232842 mutation does not necessarily have fatal consequences as evidenced by a bull homozygous for the putative disease allele without any apparent signs of disease. Although minimizing exposure to environmental pollutants and respiratory pathogens may contribute to a less severe disease progression [53], our results suggest that the mutation is highly penetrant because 17 out of 18 homozygous animals were stillborn or diseased shortly after birth. In any case, our findings now enable to implement genomebased mating strategies to avoid the mating of carrier animals thereby preventing the birth of homozygous calves that are likely to suffer from chronic disease.

\section{Conclusions}

A missense mutation in TUBD1 is associated with high juvenile mortality in Braunvieh and, unexpectedly, Fleckvieh cattle. Since genomic analyses revealed no evidence for an exchange of genetic material between both breeds, we demonstrate for the first time that the same harmful recessive mutation may segregate in different closed populations, indicating that such mutations may have occurred many generations ago. Homozygous animals suffer from chronic airway disease possibly resulting from defective cilia in the respiratory tract. Our findings now enable the implementation of genome-based mating strategies in order to prevent animal suffering and economic losses.

\section{Methods}

\section{Animals, genotypes, quality control and phasing}

Genotypes of 8501 Braunvieh and 37,314 Fleckvieh animals were extracted from the Austrian and German bovine genome databases. Genotypes of 1765 Braunvieh bulls were provided by breeding organisations from Switzerland $(n=$ 993), Italy $(n=465)$ and USA $(n=316)$ based on bilateral genotype exchange agreements. All animals had been 
genotyped with the Illumina BovineSNP50 bead chip (version 1 and version 2) comprising approximately 54,000 SNPs. The chromosomal position of the SNPs was based on the UMD 3.1 assembly of the bovine genome [54]. Mitochondrial, X-chromosomal, Y-chromosomal SNPs and SNPs that had no known chromosomal position were not considered for further analyses. Animals with a call-rate less than $95 \%$ were excluded as well as animals with mendelian conflicts with their genotyped sires at more than 200 SNPs. We excluded SNPs with a minor allele frequency below $0.5 \%$ as well as SNPs with call rate less than $90 \%$ or more than $0.5 \%$ mendelian conflicts in sire-offspring pairs. The final Braunvieh data consisted of 8,446 animals and 35,382 autosomal SNPs with an average per-individual call-rate of $99.61 \%$. The final Fleckvieh data consisted of 37,314 animals and 41,027 autosomal SNPs with an average perindividual call-rate of $99.82 \%$. Haplotypes for BTA19 were inferred and sporadically missing genotypes were imputed using the shapeit software (v2.778) [55].

\section{Mapping of $\mathrm{BH} 2$ in the Braunvieh cattle population}

A sliding window with variable size (ranging from 0.75 to $8 \mathrm{Mb}$ ) was shifted along chromosome 19 (in steps of $0.5 \mathrm{x}$ window size). Within each sliding window, the observed number of homozygotes was compared to the expected number for all haplotypes with a frequency above $3 \%$ using Fisher exact tests. The expected number of homozygous animals was calculated using haplotype information from sire, maternal grandsire and haplotype frequency. Phenotypic effects were analyzed for all haplotypes that showed significant depletion of homozygous animals $\left(P<1 \times 10^{-4}\right)$.

\section{Mapping of $\mathrm{BH}_{\mathrm{Fv}}$ in the Fleckvieh cattle population}

To identify a haplotype specific for the rs383232842 Callele, we exploited haplotypes of 10,363 Fleckvieh animals that had been genotyped and (partially) imputed at 652,853 SNPs [24]. We extracted haplotypes of 1947 animals that had also been genotyped at rs383232842 (see below). A haplotype window consisting of five adjacent SNPs was centered at rs383232842 and increased by one SNP on either side as long as a common haplotype $\left(\mathrm{BH} 2_{\mathrm{FV}}\right)$ was heterozygous in all animals that were heterozygous at rs383232842. To assess the frequency of $\mathrm{BH} 22_{\mathrm{FV}}$ in a representative sample of the Fleckvieh cattle population, we analysed haplotypes of 37,314 Fleckvieh animals from the bovine genome databases (see above).

\section{Mapping of $\mathrm{BH} 2$ in the Holstein cattle population}

To test if the common $\mathrm{BH} 2 / \mathrm{BH} 2_{\mathrm{FV}}$ haplotype also segregates in the Holstein cattle breed, we used haplotypes of 8840 Holstein artificial insemination bulls that had been genotyped previously with the Illumina BovineSNP50 Bead chip [56]. BH2 carriers were identified based on eight SNPs (from 10,694,269 bp to $11,435,269 \mathrm{bp}$ ) that were common in $\mathrm{BH} 2 / \mathrm{BH} 2_{\mathrm{FV}}$ carriers, respectively.

\section{Phenotypic effects associated with $\mathrm{BH} 2$ and $\mathrm{BH} 2_{\mathrm{FV}}$}

Insemination success was analyzed based on 55,837 artificial inseminations that were carried out with Braunvieh bulls that had a known haplotype status for $\mathrm{BH} 2$. The insemination success in risk matings (carrier bulls mated to daughters of carrier sires) was compared with nonrisk matings (non-carrier bulls mated to daughters of carrier sires) using a logistic regression model. Perinatal and first-year mortality was analyzed in 32,100 and 46,566 Braunvieh and Fleckvieh calvings, respectively, with known haplotype states from sires and maternal grandsires. Calves that left the recording system within 365 days after birth for other reasons than death (e.g., export to other countries) were not considered. A Kaplan-Meier estimator was obtained by comparing the first-year survival rate of calves from risk matings with non-risk matings [57].

\section{Generation of sequence data}

Genomic DNA of 290 animals (149 Fleckvieh, 54 Braunvieh, 51 Holstein, 15 Original Simmental, 12 Gelbvieh, 7 Northern Finncattle, 2 Ayrshire) was prepared from semen and blood samples following standard DNA extraction protocols as previously described [14]. Briefly, paired-end libraries were prepared using the paired-end TruSeq DNA sample prep kit (Illumina inc., San Diego, CA, USA) and sequenced using the Illumina HiSeq 2500 instrument (Illumina inc., San Diego, CA, USA). The resulting reads were processed with the Illumina BaseCaller during the sequencing step. The alignment of the reads to the University of Maryland reference sequence (UMD3.1) [54] was performed using the BWA mem algorithm [58, 59]. The resulting per individual SAM files were converted into BAM files with SAMtools [60]. Duplicate reads were identified and marked with the MarkDuplicates command of Picard [61]. Part of the sequencing data were previously generated $[14,62]$ and were contributed to the 1000 bull genomes project [21].

\section{Variant calling and imputation}

SNPs, short insertions and deletions were genotyped in 290 sequenced animals simultaneously using the multisample approach implemented in mpileup of SAMtools along with BCFtools [60]. Beagle [63] phasing and imputation was used to improve the primary genotype calling by SAMtools. The detection of structural variants was performed in 227 sequenced animals with an average genome fold coverage higher than $10 \mathrm{x}$ using the Pindel software package [64]. The functional effects of polymorphic sites were predicted based on the annotation of the UMD3.1 
assembly of the bovine genome using the Variant Effect Predictor tool from Ensembl $[65,66]$. The consequences of non-synonymous amino acid substitutions on protein function were predicted using PolyPhen-2 [67] and SIFT [68].

\section{Identification of $\mathrm{BH} 2$-associated variants}

We screened 110,294 polymorphic sites within a $9.71 \mathrm{Mb}$ segment on BTA19 encompassing BH2 (from 3.67 $\mathrm{Mb}$ to $13.38 \mathrm{Mb}$ ) for variants that were homozygous for the alternate allele in $\mathrm{BH} 2_{\mathrm{hom}}$, heterozygous in five $\mathrm{BH} 2$ carriers and homozygous for the reference allele in 48 control animals of the Braunvieh population. The genotype distributions of 52 variants that fulfilled these criteria were assessed in 236 sequenced animals representing six cattle breeds other than Braunvieh.

\section{Validation of the rs 383232842 polymorphism}

Genotypes for rs383232842 were obtained in 661 Braunvieh, 3807 Fleckvieh and 503 Holstein animals using a customized KASP ${ }^{\mathrm{Tx}}$ genotyping assay (LGC Genomics) (FAM/HEX/reverse primer sequence: TGTTCATGAGAA CGATGCTGTTCG/CTTGTTCATGAGAACGATGCTG TTCA/TGCTTGATATTCATCAGCTTCACACAGAT).

\section{Clinical examinations}

Seven animals were hospitalized for a period of 19 to 164 days at the animal clinic. Initial examination (including weighing) was performed upon admission. Weight records and blood samples from three animals were collected once or twice a week. Red and white blood cell count was determined using an automatic hematology analyzer (ADVIA 2120i, Siemens Healthcare, Vienna, Austria). Glucose, total protein, albumin, cholesterol, NEFA, total bilirubin, creatinine, potassium, sodium, AST, GLDH, LDH were measured in blood plasma using an automatic analyzer (Cobas 6000/c501, Roche Diagnostics $\mathrm{GmbH}$, Rotkreuz, Switzerland).

\section{Pathological examinations}

All hospitalized animals were euthanized at 32 to 502 days of age because they suffered from chronic disease with no prospect of improvement. Necropsy and collection of tissue samples were done immediately after euthanasia. For histology, samples from nasal mucosa, trachea, lung, heart, liver, kidney, pancreas, small and large intestine, mesenteric lymph node, intestine were fixed in $10 \%$ buffered formalin and embedded in paraffin wax. Sections $(3 \mu \mathrm{m})$ were stained with hematoxylin and eosin (HE) and alcian blue. For transmission electron microscopy, samples from nasal mucosa, trachea, bronchi and bronchioles were collected from four animals and fixed in $5 \%$ glutaraldehyde (Merck, Darmstadt, Germany) in $0.1 \mathrm{M}$ phosphate buffer (Sigma-Aldrich, Vienna, Austria), $\mathrm{pH} 7.2$, at $4{ }^{\circ} \mathrm{C}$ for $3 \mathrm{~h}$ and then postfixed in $1 \%$ osmium tetroxide (Merck, Darmstadt, Germany) in the same buffer at $4{ }^{\circ} \mathrm{C}$ for $2 \mathrm{~h}$. After dehydration in an alcohol gradient series and propylene oxide (Merck, Darmstadt, Germany), the tissue samples were embedded in glycidyl ether 100 (Serva, Heidelberg, Germany). The ultrathin sections were cut on a Leica Ultramicrotome (Leica Ultracut S, Vienna, Austria) and stained with uranyl acetate (Sigma-Aldrich, Vienna, Austria) and lead citrate (Merck, Darmstadt, Germany). Ultrathin sections were examined with a Zeiss TEM 900 electron microscope (Carl Zeiss, Oberkochen, Germany) operated at $50 \mathrm{kV}$.

\section{Additional files}

Additional file 1: Genotype distribution of 52 sequence variants in LD with $\mathrm{BH} 2$. Non-reference allele frequency and genotype distribution of 52 variants in LD with $\mathrm{BH} 2$ in 290 animals representing nine cohorts (number of animals homozygous for the reference allele | number of heterozygous animals | number of animals homozygous for the non-reference allele). Grey background highlights a synonymous variant in PPM1E.). Bold type indicates five variants that were located within the $1.14 \mathrm{Mb} \mathrm{BH} 2$ haplotype. Blue background indicates three variants that were not homozygous among 1682 animals that had been sequenced in the 1000 bull genomes project. Red color highlights a missense variant (rs383232842) in the TUBD1 gene. (XLSX $45 \mathrm{~kb}$ )

Additional file 2: Functional effects of 52 variants in $\mathrm{LD}$ with $\mathrm{BH} 2$. The functional consequences of the non-reference alleles were predicted using the Variant Effect Predictor tool from Ensembl. (TXT $16 \mathrm{~kb}$ )

Additional file 3: Genotypes of three sequence variants compatible with recessive inheritance of $\mathrm{BH} 2$ in 290 sequenced animals representing seven cattle breeds. The sequenced Braunvieh animals were classified into carrier and non-carrier animals using haplotypes inferred from array-derived genotypes. Blue color indicates the missense mutation in TUBD1. (PDF $34 \mathrm{~kb}$ )

Additional file 4: Across-species conservation of tubulin delta 1. Part of the multispecies alignment of the TUBD1 protein sequence. Blue color highlights the missense mutation. (PDF $73 \mathrm{~kb}$ )

Additional file 5: Genotype distribution of three variants in LD with $\mathrm{BH}$ 2. (XLSX $28 \mathrm{~kb}$ )

Additional file 6: Haplotype analysis in Braunvieh, Fleckvieh and Holstein cattle. The $\mathrm{BH} 2$ and $\mathrm{BH}_{2} \mathrm{Fv}$ haplotypes were detected using $777 \mathrm{~K}$ and $50 \mathrm{~K}$ genotyping data, respectively. Yellow and red color highlights eight common SNPs in Braunvieh and Fleckvieh cattle and the missense variant in TUBD1, respectively. Note that the $\mathrm{BH} 2$ haplotype version in Holstein cattle does not contain the rs383232842 C-allele. (XLSX $62 \mathrm{~kb}$ )

Additional file 7: Cluster analyses in 280 Braunvieh and 442 Fleckvieh animals. Principal component analysis (PCA) using genotypes of 42,056 autosomal SNPs (a) and 1121 SNPs located on chromosome 19 (b), respectively. All animals were born between 1970 and 2010. The PCA separated the animals by breed without any evidence for an admixture. There was no indication that $\mathrm{BH} 2$ and $\mathrm{BH}_{2} \mathrm{Fv}$ carriers were more closely related to each other than to non-carrier animals. Hierarchical clustering using genotypes of 1121 SNPs located on chromosome 19 (c). Animals were clearly separated by breed without any evidence of an admixture of $\mathrm{BH} 2$ and $\mathrm{BH}_{2} \mathrm{FV}$ carriers. (TIF $3541 \mathrm{~kb}$ )

Additional file 8: Photograph of a stillborn homozygous calf. Despite a normal gestation length (283 days), the calf was underweight at birth at $25 \mathrm{~kg}$. The head of the stillborn calf appears elongated and the limbs were thin and elongated. The analysis of histological sections of a diverse tissue panel revealed no pathological findings. (PNG $1867 \mathrm{~kb}$ )

Additional file 9: Growth of six $\mathrm{BH} 2$ homozygous calves. The weight of six $\mathrm{BH} 2$ homozygous calves (BV1-5, FV1) at admission to the clinic and at 
euthanasia is compared to 74,422 healthy Fleckvieh animals (grey boxes) and three Fleckvieh animals with loss of function (LoF) variants in GON4L and SLC2A2 that manifest in growth retardation [14, 22]. (TIF $180 \mathrm{~kb}$ )

Additional file 10: Blood parameters of BV1, BV2 and FV1. The grey shaded areas represent reference values that were determined based on [69]. (TIF $2443 \mathrm{~kb}$ )

Additional file 11: Macroscopic lesions in the respiratory tract in four homozygous animals. Congestion and mucopurulent inflammation of the nasal conchae of BV2 (a). Different grades of obstruction, atelectasis and/ or bronchopneumonia (b-d): single consolidated area in the right cranial lung lobe of BV1 (b), multiple lobular consolidations in both cranial and middle lung lobes of FV1 (c) and severe pneumonic induration of the majority of both cranial and middle lung lobes of BV5 (d). Arrows indicate the border to physiological lung tissue. (PNG $1127 \mathrm{~kb}$ )

Additional file 12: Histological lesions in the respiratory tract of two homozygous animals. The ciliated epithelium of the nasal mucosa and the underlying submucosa of FV1 are infiltrated with inflammatory cells, particularly neutrophils (a). Lung lesions of BV5 including hyperplastic bronchiolar epithelium, partial obstruction of the bronchiolar lumen by purulent exsudate and consolidation of the surrounding lung tissue due to obstructive atelectasis and mild infiltration with neutrophils (b). (PNG $720 \mathrm{~kb}$ )

Additional file 13: Three animals homozygous for the rs383232842 Callele. A homozygous young bull (BV6) at the age of 370 days (a). According to the possessing farmer, the bull did not suffer from respiratory disease in the past. The weight of another homozygous young bull (BV7) at the age of 271 days was $243 \mathrm{~kg}$ (b). The possessing farmer reported that the young bull suffered from life-threatening bronchopneumonia early in life. Although the animal recovered from the disease, coughing and excessive mucous exudation from the nostrils were apparent during its entire life. Multiple lung lesions were noticed after slaughter. A female calf homozygous (BV8) for the rs383232842 C-allele at the age of 194 days with an unaffected coeval (c-e). Photographs were kindly provided by Franz Birkenmaier (a, c-e) and Attilio Rossoni (b). (TIF $5396 \mathrm{~kb}$ )

\section{Abbreviations}

Al, artificial insemination; BALT, bronchus-associated lymphoid tissue; $\mathrm{BH} 2$ Braunvieh haplotype 2; BV, Braunvieh; FV, Fleckvieh; LD, linkage disequilibrium; SNP, Single nucleotide polymorphism; TEM, transmission electron microscopy

\section{Acknowledgements}

We acknowledge the InterGenomics partners (Arbeitsgemeinschaft der Österreichischen Braunviehzüchter, Austria; Arbeitsgemeinschaft Deutsches Braunvieh, Germany; Associazione Nazionle Allevatori Bovini della Razza Bruna Italiana, Italy; Brown Swiss Association of the US, USA; Brune Genetique Services, France; Braunvieh Schweiz, Switzerland; and Zveza rejcev govedi rjave pasme Slovenije, Slovenia), the Arbeitsgemeinschaft Süddeutscher Rinderzüchter und Besamungsorganisationen e.V. (ASR), the Arbeitsgemeinschaft österreichischer Fleckviehzüchter (AGÖF) and the Förderverein Bioökonomieforschung e.V. (FBF) for providing genotype data. We thank Associazione Nazionale Allevatori Bovini della Razza Bruna Italiana (ANARBI) and Arbeitsgemeinschaft Deutsches Braunvieh for providing pictures of affected animals and support in sample collection.

\section{Funding}

The calf monitoring project was financially supported by Braunvieh Austria and Braunvieh Schweiz. The funders had no role in study design, data collection and analysis, decision to publish, or preparation of the manuscript.

\section{Availability of supporting data}

Whole-genome sequencing data of $\mathrm{BH} 2_{\text {hom }}$ were deposited in the European Nucleotide Archive under accession number PRJEB12807 (http://www.ebi.ac.uk/ ena/data/view/PRJEB12807). The sequencing data of 241 animals from the 1000 bull genomes project are publically available in the Sequence Read Archive of NCBI (http://www.ncbi.nlm.nih.gov/sra) under accession number SRP039339.

\section{Authors' contributions}

$\mathrm{HP}, \mathrm{HS}$ and RF conceived the study, designed the experiments and analyzed the data; HS and CF analyzed genotype and phenotype data; HP analyzed sequence and genotype data; HS, FRS, RW and OG conceived the calf monitoring project; JB, TW, ND, MH, HW and UB examined homozygous animals at the animal clinic; CW carried out the sequencing experiments, CW and SJ carried out the molecular genetic experiments, BFW and MD contributed to the design of the experiment, HP wrote the manuscript.

\section{Competing interests}

The authors declare that they have no competing interests.

\section{Consent for publication}

Not applicable.

\section{Ethics approval and consent to participate}

All homozygous animals examined were descendants from matings between carrier animals that happened inadvertently in the Braunvieh and Fleckvieh populations prior to the declaration of $\mathrm{BH} 2$ carrier bulls. Ear tissue samples of calves from at-risk matings were collected from trained breeding consultants. Seven diseased homozygous animals were hospitalized and examined at the Clinic for Ruminants of the Veterinary University of Vienna, Austria and at the Clinic of Reproductive Medicine, Vetsuisse-Faculty, University Zurich, Switzerland as part of their regular practice. All hospitalized animals were euthanized because of steadily declining health condition resulting from recurrent airway disease with no prospect of improvement. The decision to euthanize the animals was at the discretion of the veterinarians at the animal clinic. DNA of healthy animals for genotyping and sequencing was prepared from semen samples of artificial insemination (Al) bulls. Semen samples were collected by approved commercial Al stations as part of their regular breeding and reproduction measures in cattle industry. No ethical approval was required for this study.

\section{Author details}

'ZuchtData EDV Dienstleistungen $\mathrm{GmbH}$, Vienna 1200, Austria. ${ }^{2} \mathrm{Clinic}$ for Ruminants, University of Veterinary Medicine Vienna, Vienna 1210, Austria. ${ }^{3}$ Qualitas AG, Zug 6300, Switzerland. ${ }^{4}$ Lehrstuhl fuer Tierzucht, Technische Universitaet Muenchen, Freising 85354, Germany. ${ }^{5}$ Institute of Veterinary Pathology, Vetsuisse-Faculty, University Zurich, Zurich 8057, Switzerland. ${ }^{6}$ Institute of Pathology and Forensic Veterinary Medicine, University of Veterinary Medicine, Vienna, Austria. ${ }^{7}$ Division of Livestock Sciences, University of Natural Resources and Life Sciences, Vienna, Austria. ${ }^{8}$ Platform Bioinformatics and Statistics, University of Veterinary Medicine, Vienna, Austria. ${ }^{9}$ ARGE Braunvieh, Innsbruck 6020, Austria. ${ }^{10}$ Braunvieh Schweiz, Zug 6300, Switzerland. ${ }^{11}$ Clinic of Reproductive Medicine, Department of Farm Animals, Vetsuisse-Faculty, University Zurich, Zurich 8057, Switzerland.

Received: 1 March 2016 Accepted: 14 May 2016

Published online: 25 May 2016

\section{References}

1. Gulliksen SM, Lie Kl, Løken T, Osterås O. Calf mortality in Norwegian dairy herds. J Dairy Sci. 2009;92:2782-95.

2. Silva del Río N, Stewart S, Rapnicki P, Chang YM, Fricke PM. An observational analysis of twin births, calf sex ratio, and calf mortality in Holstein dairy cattle. J Dairy Sci. 2007;90:1255-64.

3. Brickell JS, McGowan MM, Pfeiffer DU, Wathes DC. Mortality in HolsteinFriesian calves and replacement heifers, in relation to body weight and IGF-I concentration, on 19 farms in England. Animal. 2009:3:1175-82.

4. Fuerst-Waltl B, Fuerst C. Effect of inbreeding depression on survival of Austrian Brown Swiss calves and heifers. J Dairy Sci. 2012;95:6086-92.

5. Windeyer MC, Leslie KE, Godden SM, Hodgins DC, Lissemore KD, LeBlanc SJ. Factors associated with morbidity, mortality, and growth of dairy heifer calves up to 3 months of age. Prev Vet Med. 2014;113:231-40.

6. Olsen HG, Hayes BJ, Kent MP, Nome T, Svendsen M, Lien S. A genome wide association study for QTL affecting direct and maternal effects of stillbirth and dystocia in cattle. Anim Genet. 2010;41:273-80.

7. Pausch H, Flisikowski K, Jung S, Emmerling R, Edel C, Götz K-U, et al. Genome-Wide Association Study Identifies Two Major Loci Affecting Calving Ease and Growth-Related Traits in Cattle. Genetics. 2011;187:289-97.

8. Hansen M, Madsen P, Jensen J, Pedersen J, Christensen LG. Genetic parameters of postnatal mortality in Danish Holstein calves. J Dairy Sci. 2003;86:1807-17.

9. Fuerst-Waltl B, Sørensen MK Genetic analysis of calf and heifer losses in Danish Holstein. J Dairy Sci. 2010;93:5436-42. 
10. Jung S, Pausch H, Langenmayer MC, Schwarzenbacher H, Majzoub-Altweck M, Gollnick NS, et al. A nonsense mutation in PLD4 is associated with a zinc deficiency-like syndrome in Fleckvieh cattle. BMC Genomics. 2014;15:623.

11. Sartelet A, Druet T, Michaux C, Fasquelle C, Géron S, Tamma N, et al. A Splice Site Variant in the Bovine RNF11 Gene Compromises Growth and Regulation of the Inflammatory Response. PLoS Genet. 2012;8, e1002581.

12. Hirano T, Kobayashi N, Matsuhashi T, Watanabe D, Watanabe T, Takasuga A, et al. Mapping and Exome Sequencing Identifies a Mutation in the IARS Gene as the Cause of Hereditary Perinatal Weak Calf Syndrome. PLoS One. 2013;8, e64036.

13. Venhoranta H, Pausch H, Flisikowski K, Wurmser C, Taponen J, Rautala H, et al. In frame exon skipping in UBE3B is associated with developmental disorders and increased mortality in cattle. BMC Genomics. 2014;15:890.

14. Pausch $\mathrm{H}$, Schwarzenbacher $\mathrm{H}$, Burgstaller J, Flisikowski K, Wurmser $\mathrm{C}$, Jansen $\mathrm{S}$, et al. Homozygous haplotype deficiency reveals deleterious mutations compromising reproductive and rearing success in cattle. BMC Genomics. 2015:16:312.

15. Kipp S, Segelke D, Reinhardt F, Reents R, Schierenbeck S, Wurmser C, et al. A new Holstein Haplotype affecting calf survival. Interbull Bulletin. 2015;49:49-53.

16. Menzi F, Besuchet-Schmutz N, Fragnière M, Hofstetter S, Jagannathan V, Mock T, et al. A transposable element insertion in APOB causes cholesterol deficiency in Holstein cattle. Anim Genet. 2016;47:253-7.

17. VanRaden PM, Olson KM, Null DJ, Hutchison JL. Harmful recessive effects on fertility detected by absence of homozygous haplotypes. J Dairy Sci. 2011;94:6153-61.

18. Fritz S, Capitan A, Djari A, Rodriguez SC, Barbat A, Baur A, et al. Detection of Haplotypes Associated with Prenatal Death in Dairy Cattle and Identification of Deleterious Mutations in GART, SHBG and SLC37A2. PLoS One. 2013;8, e65550.

19. Schwarzenbacher H, Fuerst C, Fuerst-Waltl B, Dolezal M. A genome-wide search for harmful recessive haplotypes in Brown Swiss and Fleckvieh cattle. In: Book of Abstracts of the 63rd European Association for Animal Production Annual Meeting Bratislava. Slovakia: EAAP; 2012.

20. AIPL Changes to Evaluation System (August 2013). https://www.cdcb.us/ reference/changes/eval1308.htm. Accessed 6 April 2016

21. Daetwyler HD, Capitan A, Pausch $H$, Stothard $P$, van Binsbergen $R$, Brøndum RF, et al. Whole-genome sequencing of 234 bulls facilitates mapping of monogenic and complex traits in cattle. Nat Genet. 2014;46:858-65.

22. Schwarzenbacher $H$, Wurmser C, Flisikowski K, Misurova L, Jung S, Langenmayer MC, et al. A frameshift mutation in GON4L is associated with proportionate dwarfism in Fleckvieh cattle. Genet Sel Evol. 2016:48:25.

23. Drögemüller $C$, Reichart $U$, Seuberlich T, Oevermann A, Baumgartner $M$, Kühni Boghenbor K, et al. An Unusual Splice Defect in the Mitofusin 2 Gene (MFN2) Is Associated with Degenerative Axonopathy in Tyrolean Grey Cattle. PLoS One. 2011;6, e18931.

24. Pausch H, Kölle S, Wurmser C, Schwarzenbacher H, Emmerling R, Jansen S, et al. A Nonsense Mutation in TMEM95 Encoding a Nondescript Transmembrane Protein Causes Idiopathic Male Subfertility in Cattle. PLoS Genet. 2014;10, e1004044.

25. Kadri NK, Sahana G, Charlier C, Iso-Touru T, Guldbrandtsen B, Karim L, et al, A 660-Kb Deletion with Antagonistic Effects on Fertility and Milk Production Segregates at High Frequency in Nordic Red Cattle: Additional Evidence for the Common Occurrence of Balancing Selection in Livestock. PLoS Genet. 2014;10, e1004049.

26. Drögemüller C, Tetens J, Sigurdsson S, Gentile A, Testoni S, Lindblad-Toh K, et al. Identification of the Bovine Arachnomelia Mutation by Massively Parallel Sequencing Implicates Sulfite Oxidase (SUOX) in Bone Development. PLoS Genet. 2010;6, e1001079.

27. Thomsen B, Nissen PH, Agerholm JS, Bendixen C. Congenital bovine spinal dysmyelination is caused by a missense mutation in the SPAST gene. Neurogenetics. 2010;11:175-83.

28. Krebs S, Medugorac I, Röther S, Strässer K, Förster M. A missense mutation in the 3-ketodihydrosphingosine reductase FVT1 as candidate causal mutation for bovine spinal muscular atrophy. Proc Natl Acad Sci U S A. 2007:104:6746-51.

29. McClure MC, Bickhart D, Null D, VanRaden P, Xu L, Wiggans G, et al. Bovine Exome Sequence Analysis and Targeted SNP Genotyping of Recessive Fertility Defects $\mathrm{BH} 1, \mathrm{HH} 2$, and $\mathrm{HH} 3$ Reveal a Putative Causative Mutation in SMC2 for HH3. PLoS One. 2014;9, e92769.

30. Biffani S, Dimauro C, Macciotta N, Rossoni A, Stella A, Biscarini F. Predicting haplotype carriers from SNP genotypes in Bos taurus through linear discriminant analysis. Genet Sel Evol. 2015;47:4.
31. Drögemüller $M$, Jagannathan $V$, Becker $D$, Drögemüller $C$, Schelling $C$, Plassais J, et al. A Mutation in the FAM83G Gene in Dogs with Hereditary Footpad Hyperkeratosis (HFH). PLoS Genet. 2014;10, e1004370.

32. McClure M, Kim E, Bickhart D, Null D, Cooper T, Cole J, et al. Fine Mapping for Weaver Syndrome in Brown Swiss Cattle and the Identification of 41 Concordant Mutations across NRCAM, PNPLA8 and CTTNBP2. PLoS One. 2013;8, e59251.

33. Kunz E, Rothammer S, Pausch H, Schwarzenbacher H, Seefried FR, Matiasek $\mathrm{K}$, et al. Confirmation of a non-synonymous SNP in PNPLA8 as a candidate causal mutation for Weaver syndrome in Brown Swiss cattle. Genet Sel Evol. 2016:48:21.

34. Decker JE, McKay SD, Rolf MM, Kim J, Molina Alcalá A, Sonstegard TS, et al. Worldwide Patterns of Ancestry, Divergence, and Admixture in Domesticated Cattle. PLoS Genet. 2014;10, e1004254.

35. Noone PG, Leigh MW, Sannuti A, Minnix SL, Carson JL, Hazucha M, et al. Primary Ciliary Dyskinesia. Am J Respir Crit Care Med. 2004;169:459-67.

36. Coren M, Meeks M, Morrison I, Buchdahl R, Bush A. Primary ciliary dyskinesia: age at diagnosis and symptom history. Acta Paediatr. 2002;91:667-9.

37. Leigh MW, Pittman JE, Carson JL, Ferkol TW, Dell SD, Davis SD, et al. Clinical and genetic aspects of primary ciliary dyskinesia/Kartagener syndrome. Genet Med. 2009:11:473-87.

38. Wisseman CL, Simel DL, Spock A, Shelburne JD. The prevalence of abnormal cilia in normal pediatric lungs. Arch Pathol Lab Med. 1981;105:552-5.

39. Chilvers M, Rutman A, O'Callaghan C. Functional analysis of cilia and ciliated epithelial ultrastructure in healthy children and young adults. Thorax. 2003:58:333-8.

40. Ziętkiewicz E, Bukowy-Bieryłło Z, Voelkel K, Klimek B, Dmeńska H, Pogorzelski A, et al. Mutations in Radial Spoke Head Genes and Ultrastructural Cilia Defects in East-European Cohort of Primary Ciliary Dyskinesia Patients. PLoS One. 2012;7, e33667.

41. Carson JL, Collier AM, Hu SS. Acquired ciliary defects in nasal epithelium of children with acute viral upper respiratory infections. N Engl J Med. 1985; 312:463-8.

42. Zariwala MA, Knowles MR, Omran H. Genetic Defects in Ciliary Structure and Function. Annu Rev Physiol. 2007;69:423-50

43. Chilvers MA, Rutman A, O'Callaghan C. Ciliary beat pattern is associated with specific ultrastructural defects in primary ciliary dyskinesia. J Allergy Clin Immunol. 2003;112:518-24.

44. Leigh MW, O'Callaghan C, Knowles MR. The Challenges of Diagnosing Primary Ciliary Dyskinesia. Proc Am Thorac Soc. 2011;8:434-7.

45. Olm MAK, Kögler JE, Macchione M, Shoemark A, Saldiva PHN, Rodrigues JC. Primary ciliary dyskinesia: evaluation using cilia beat frequency assessment via spectral analysis of digital microscopy images. J Appl Physiol. 2011;111:295-302.

46. Merveille A-C, Davis EE, Becker-Heck A, Legendre M, Amirav I, Bataille G, et al. CCDC39 is required for assembly of inner dynein arms and the dynein regulatory complex and for normal ciliary motility in humans and dogs. Nat Genet. 2011;43:72-8.

47. Lee L, Campagna DR, Pinkus JL, Mulhern H, Wyatt TA, Sisson JH, et al. Primary ciliary dyskinesia in mice lacking the novel ciliary protein Pcdp1. Mol Cell Biol. 2008:28:949-57.

48. Dutcher SK, Trabuco EC. The UNI3 Gene Is Required for Assembly of Basal Bodies of Chlamydomonas and Encodes $\delta$-Tubulin, a New Member of the Tubulin Superfamily. Mol Biol Cell. 1998;9:1293-308.

49. Inclán YF, Nogales E. Structural models for the self-assembly and microtubule interactions of gamma-, delta- and epsilon-tubulin. J Cell Sci. 2001;114:413-22.

50. OToole ET, Giddings TH, McIntosh JR, Dutcher SK. Three-dimensional Organization of Basal Bodies from Wild-Type and $\delta$-Tubulin Deletion Strains of Chlamydomonas reinhardtii. Mol Biol Cell. 2003;14:2999-3012.

51. Garreau de Loubresse N, Ruiz F, Beisson J, Klotz C. Role of delta-tubulin and the C-tubule in assembly of Paramecium basal bodies. BMC Cell Biol. 2001;2:4.

52. Heald R, Khodjakov A. Thirty years of search and capture: The complex simplicity of mitotic spindle assembly. J Cell Biol. 2015;211:1103-11.

53. Barbato A, Frischer T, Kuehni CE, Snijders D, Azevedo I, Baktai G, et al. Primary ciliary dyskinesia: a consensus statement on diagnostic and treatment approaches in children. Eur Respir J. 2009;34:1264-76.

54. Zimin AV, Delcher AL, Florea L, Kelley DR, Schatz MC, Puiu D, et al. A wholegenome assembly of the domestic cow. Bos taurus Genome Biol. 2009;10:R42.

55. Delaneau O, Zagury J-F, Marchini J. Improved whole-chromosome phasing for disease and population genetic studies. Nat Meth. 2013;10:5-6. 
56. Pausch H, Wurmser C, Reinhardt F, Emmerling R, Fries R. Short communication: Validation of 4 candidate causative trait variants in 2 cattle breeds using targeted sequence imputation. J Dairy Sci. 2015;98:4162-7.

57. Therneau TM, Grambsch PM. Modeling survival data: extending the Cox model. New York: Springer; 2000.

58. Li H. Aligning sequence reads, clone sequences and assembly contigs with BWA-MEM. Preprint at http://arxiv.org/pdf/1303.3997.pdf. 2013. Accessed 24 May 2016

59. Li H, Durbin R. Fast and accurate short read alignment with BurrowsWheeler transform. Bioinformatics. 2009;25:1754-60.

60. Li H, Handsaker B, Wysoker A, Fennell T, Ruan J, Homer N, et al. The Sequence Alignment/Map format and SAMtools. Bioinformatics. 2009;25:2078-9.

61. Picard Tools - By Broad Institute: http://broadinstitute.github.io/picard/. Accessed 21 October 2015

62. Jansen $\mathrm{S}$, Aigner B, Pausch $\mathrm{H}$, Wysocki M, Eck S, Benet-Pagès A, et al. Assessment of the genomic variation in a cattle population by resequencing of key animals at low to medium coverage. BMC Genomics. 2013;14:446.

63. Browning BL, Browning SR. A Unified Approach to Genotype Imputation and Haplotype-Phase Inference for Large Data Sets of Trios and Unrelated Individuals. Am J Hum Genet. 2009:84:210-23.

64. Ye K, Schulz MH, Long Q, Apweiler R, Ning Z. Pindel: a pattern growth approach to detect break points of large deletions and medium sized insertions from paired-end short reads. Bioinformatics. 2009;25:2865-71.

65. McLaren W, Pritchard B, Rios D, Chen Y, Flicek P, Cunningham F. Deriving the consequences of genomic variants with the Ensembl API and SNP Effect Predictor. Bioinformatics. 2010;26:2069-70.

66. Variant Effect Predictor. http://www.ensembl.org/Tools/NEP. Accessed 13 January 2016

67. Adzhubei IA, Schmidt S, Peshkin L, Ramensky VE, Gerasimova A, Bork P, et al. A method and server for predicting damaging missense mutations. Nat Methods. 2010;7:248-9.

68. Ng PC, Henikoff S. SIFT: predicting amino acid changes that affect protein function. Nucl Acids Res. 2003:31:3812-4.

69. Kraft W, Dürr UM. Klinische Labordiagnostik in der Tiermedizin. 6th ed. Stuttgart: Schattauer Verlag; 2005.

\section{Submit your next manuscript to BioMed Central and we will help you at every step:}

- We accept pre-submission inquiries

- Our selector tool helps you to find the most relevant journal

- We provide round the clock customer support

- Convenient online submission

- Thorough peer review

- Inclusion in PubMed and all major indexing services

- Maximum visibility for your research

Submit your manuscript at www.biomedcentral.com/submit 